DRAFT VERSiON JANUARY 20, 2020

Preprint typeset using LATEX style emulateapj v. 01/23/15

\title{
TRANSIENT X-RAY SOURCE POPULATION IN THE MAGELLANIC-TYPE GALAXY NGC 55
}

\author{
V. Jithesh AND ZHONGXIANG WANG \\ Shanghai Astronomical Observatory, Chinese Academy of Sciences, \\ 80 Nandan Road, Shanghai 200030, China; jithesh@shao.ac.cn \\ Draft version January 20, 2020
}

\begin{abstract}
We present the spectral and temporal properties of 15 candidate transient X-ray sources detected in archival XMM-Newton and Chandra observations of the nearby Magellanic-type, SB(s)m galaxy NGC 55. On the basis of an X-ray color classification scheme, the majority of the sources may be identified as X-ray binaries (XRBs), and six sources are soft, including a likely supernova remnant. We perform a detailed spectral and variability analysis of the data for two bright candidate XRBs. Both sources displayed strong short-term X-ray variability, and their X-ray spectra and hardness ratios are consistent with those of XRBs. These results, combined with their high X-ray luminosities $\left(\sim 10^{38} \mathrm{erg} \mathrm{s}^{-1}\right)$, strongly suggest that they are black hole $(\mathrm{BH})$ binaries. Seven less luminous sources have spectral properties consistent with those of neutron star or BH XRBs in both normal and highrate accretion modes, but one of them is the likely counterpart to a background galaxy (because of positional coincidence). From the spectral analysis, we find that the six soft sources are candidate super soft sources (SSSs), with dominant emission in the soft (0.3-2 keV) X-ray band. Archival Hubble Space Telescope optical images for 7 sources are available, and the data suggest that most of them are likely to be high-mass XRBs. Our analysis has revealed the heterogeneous nature of the transient population in NGC 55 (6 high-mass XRBs, 1 low-mass XRBs, 6 SSSs, 1 active galactic nucleus), helping establish the similarity of the X-ray properties of this galaxy to those of other Magellanictype galaxies.

Subject headings: galaxies : individual (NGC 55) — (galaxies:) Magellanic Clouds — X-rays: general - X-rays: binaries - X-rays: galaxies
\end{abstract}

\section{INTRODUCTION}

X-ray transients are an interesting class of sources, with quiescent luminosities below the detection limit, that are primarily discovered when they enter outbursts typically lasting from weeks to a few months. These bright outbursts are characterized by an episode of high accretion rates and abrupt increases of X-ray luminosity by several orders of magnitude. We now know that most of them are binary systems with a white dwarf (WD), a neutron star (NS), or a black hole (BH) as the accreting compact object. Monitoring observations at X-ray wavelengths have revealed a variety of spectral and temporal behavior of these sources, and established distinct X-ray spectral states for X-ray binaries, in which the compact primary is either a NS or a $\mathrm{BH}$ (see McClintock \& Remillard 2006; Remillard \& McClintock 2006, for extensive reviews on spectral states).

$\mathrm{X}$-ray transient events in the nearby galaxies originate from different types of objects, which include ultraluminous X-ray sources (ULXs), super soft sources (SSSs), low-mass X-ray binaries (LMXBs) and high-mass X-ray binaries (HMXBs). Most of the ULXs identified in the nearby galaxies are persistent sources with X-ray luminosities $\left(>10^{39} \mathrm{erg} \mathrm{s}^{-1}\right.$ ) exceeding the Eddington limit for a $10 \mathrm{M}_{\odot} \mathrm{BH}$ (see Feng \& Soria 2011, for a review). Extensive monitoring observations with $X M M$ Newton, Chandra, and Swift identified two ULX transients in M31 (Kaur et al. 2012; Barnard et al. 2013). Multi-wavelength analysis suggests that the underlying sources are likely stellar-mass BHs with low mass donors (Nooraee et al. 2012; Barnard et al. 2013). Another important class of X-ray sources in the external galaxies are SSSs, usually characterized by values of $k T$ in the range of tens of $\mathrm{eV}$, with $\mathrm{X}$-ray luminosities between $10^{36}$ and $10^{38} \mathrm{erg} \mathrm{s}^{-1}$ (Di Stefano \& Kong 2003). They were first identified in the Large Magellanic Cloud (LMC; Long et al. 1981). A promising explanation for SSSs is the quasi-steady nuclear burning on the surface of a WD, in which the compact object accretes at a high rate from a Roche lobe-filling companion van den Heuvel et al. 1992). A sizable population of SSSs are identified in the external galaxies, for example M31, M101, M83, M51 and NGC 4697 Di Stefano \& Kong 2004; Stiele et al. 2011), and some of these sources are subdivided into a new class called quasi-soft sources (QSSs; Di Stefano \& Kong 2004). QSSs are sources with little or no emission above $2 \mathrm{keV}$, with temperatures significantly higher than that of the typical SSSs, generally between $100 \mathrm{eV}$ and $350 \mathrm{eV}$. Detailed studies of SSSs revealed that they are detected in both early and latetype galaxies, identified in the field and globular clusters of a host galaxy, with a variety of temporal properties (Di Stefano \& Kong 2003; Di Stefano et al. 2004; Jenkins et al. 2005; Brassington et al. 2012). Finally, there will be possible contamination from background active galactic nuclei (AGN) in the X-ray source population of a galaxy, which are misidentified as the sources in the host galaxy Gutiérrez \& López-Corredoira 2007; Gutiérrez 2013).

NGC 55 is a Magellanic-type, SB(s)m galaxy, a member of the nearby Sculptor group of galaxies. The first observation of NGC 55 at X-ray wavelengths by ROSAT (Read et al. 1997; Schlegel et al. 1997; Dahlem et al. 1998) identified 15 discrete X-ray sources in the $\mathrm{D}_{25}$ el- 
lipse of the galaxy. A detailed analysis of X-ray properties of NGC 55 was presented by Stobbart et al. (2006, hereafter SRW06) using the XMM-Newton observations. They identified $42 \mathrm{X}$-ray sources within the optical confines of the galaxy and classified them as X-ray binaries (XRBs), supernova remnants (SNRs), and SSSs. A ULX, with X-ray luminosity $>10^{39} \mathrm{erg} \mathrm{s}^{-1}$, is also present in this galaxy, which showed a significant variability with pronounced dips and a flux increase in the light curves during the XMM-Newton observations (Stobbart et al. 2004). However, from the Swift monitoring campaign and Chandra observations, Pintore et al. (2015) reported marginal evidence for limited dips in the light curves. Recently, Binder et al. (2015, hereafter BWF15) presented a comprehensive study of X-ray point sources in three galaxies, which includes NGC 55, as a part of the Chandra Local Volume Survey (CLVS). The purpose of the CLVS was to identify the strong XRB candidates in nearby galaxies and compare the properties of the XRB population to the star formation histories of the host galaxies. They identified 154 X-ray sources in NGC 55 using multiple archival Chandra observations and studied the long-term variability using two XMM-Newton observations conducted in 2001. The X-ray hardness ratios, spectral properties, and temporal variability of the point sources were reported and tentative classification of these sources was provided as well.

As approximately one third of the known Galactic XRBs show evidence of being X-ray transients (Tanaka \& Shibazaki 1996) but the extragalactic X-ray transient population has been relatively little studied (except in M31), we have carried out studies of the transient X-ray source population in NGC 55 . Such studies for the external galaxies can give a better understanding of the nature and evolution of these sources. In this paper, we present our analysis of a long, $127 \mathrm{ks} X M M$ Newton observation conducted in 2010 along with the other archival X-ray observations (also used in BWF15). The spectral and temporal properties of transient X-ray sources identified in NGC 55 are reported. Using the Hubble Space Telescope (HST) observations and other multi-wavelength data, we address the possible nature of these sources. The paper is structured as follows. In $\S 2$, we describe the observations used and data reduction. We present the source selection and an overview of the source properties in $\S 3$. In $\S 4$, we discuss the results and possible nature of these sources, and in $\S 5$ we summarize our results.

\section{OBSERVATIONS AND DATA REDUCTION}

XMM-Newton observed NGC 55 three times: on 2001 November 14, 15, and 2010 May 25 for exposure times of $33.6,31.5$, and $127.4 \mathrm{ks}$, respectively. The observations used in this work are summarized in Table 1. The European Photon Imaging Camera (EPIC) PN (Strüder et al. 2001) and metal oxide semiconductor (MOS; Turner et al. 2001) camera were operated in the full frame mode using the thin optical blocking filter in the 2001 and 2010 observations. EPIC-PN and MOS data were reduced using standard tools (EPCHAIN and EMCHAIN respectively) of XMM-Newton Science Analysis Software (SAS), version 14.0. The full-field background light curve was extracted to remove the particle flaring background. The good time intervals (GTI)
TABLE 1

OBSERVATION LOG

\begin{tabular}{|c|c|c|c|}
\hline Data & Obs ID & Date & Exposure Time ${ }^{a}$ \\
\hline XMM1 & 0028740201 & 2001 Nov 14 & 33.6 \\
\hline XMM2 & 0028740101 & 2001 Nov 15 & 31.5 \\
\hline XMM3 & 0655050101 & 2010 May 25 & 127.4 \\
\hline Chandra & 2255 & 2001 Sep 11 & 60.1 \\
\hline Chandra & 4744 & 2004 Jun 29 & 9.7 \\
\hline HST DISK & 9765 & 2003 Dec 16 & $\begin{array}{l}0.7(\mathrm{~F} 814 \mathrm{~W}) \\
0.7(\mathrm{~F} 606 \mathrm{~W})\end{array}$ \\
\hline HST FIELD & 9765 & 2003 Sep 23 & $\begin{array}{l}0.7(\mathrm{~F} 814 \mathrm{~W}) \\
0.4(\mathrm{~F} 606 \mathrm{~W})\end{array}$ \\
\hline$H S T$ WIDE-1 & 11307 & 2007 Jul 28 & $\begin{array}{l}2.6(\mathrm{~F} 814 \mathrm{~W}) \\
0.9(\mathrm{~F} 606 \mathrm{~W})\end{array}$ \\
\hline$H S T$ WIDE-3 & 11307 & 2007 Aug 07 & $\begin{array}{l}3.9(\mathrm{~F} 814 \mathrm{~W}) \\
2.7(\mathrm{~F} 606 \mathrm{~W})\end{array}$ \\
\hline$H S T$ WIDE-4 & 11307 & 2007 Aug 09 & $\begin{array}{l}3.9(\mathrm{~F} 814 \mathrm{~W}) \\
2.7(\mathrm{~F} 606 \mathrm{~W})\end{array}$ \\
\hline$H S T$ WIDE-5 & 11307 & 2007 Aug 06 & $\begin{array}{l}3.9(\mathrm{~F} 814 \mathrm{~W}) \\
2.7(\mathrm{~F} 606 \mathrm{~W})\end{array}$ \\
\hline
\end{tabular}

Note. $-{ }^{a}$ Exposure time is in units of kiloseconds.

were generated using periods with count rate $\leq 0.8$ and $\leq 0.3 \mathrm{ct} \mathrm{s}^{-1}$ in the $10-12 \mathrm{keV}$ light curve for $\mathrm{PN}$ and MOS data respectively. We selected the events corresponding to patterns $0-4$ from the PN data and patterns 0-12 from the MOS data for the analysis with (FLAG $==0)$.

The source detection routine was carried out in the high sensitive EPIC-PN data over the entire energy band. The SAS task EBOXDETECT was used to perform the initial source detection (with a detection threshold 'likemin'=8), employing a sliding box detection method. We provided the obtained source positions as input for the task ESPLINEMAP that constructs background maps using source-free regions of the image. The EBOXDETECT task was performed again in 'map' mode using the available background map, which improved detection sensitivity. We ran the EMLDETECT task using these improved background maps with a minimum detection likelihood value of $10(\mathrm{mlmin}=10)$. If this value is 10 or greater, the sources were classified as significant detection and included in the final source list. Using the same criteria, we obtained the final source list from the 2001 and 2010 observations. The X-ray sources in the final list were then astrometrically corrected by correlating with the USNO A2.0 optical catalog (Monet 1998), in which the SAS task EPOSCORR was used.

We also analyzed two archival Chandra observations of NGC 55, conducted in 2001 September 11 and 2004 June 29 with the Advanced CCD Imaging Spectrometer Imaging Array (ACIS-I). However, the second observation was too short and none of any transient sources were detected in it. Thus we report no further analysis of this observation in the paper. The Chandra data were reduced and reprocessed using the science threads of Chandra Interactive Analysis of Observations software package (CIAO) version 4.6 and HEASOFT version 6.15.1.

In addition, we checked the archival Swift X-Ray Telescope (XRT; Burrows et al. 2005) observations for possible detection of the sources reported in this paper. However the sources, except T1 (See Jithesh \& Wang 2015, hereafter JW15), were too faint to be detected with the Swift XRT telescope.

The Optical analysis was carried out using the six 
HST fields (See Table 1 for details). We searched the candidate optical counterparts of transient sources after performing an astrometric calibration of the X-ray and HST images using the bright point sources from the Two Micron All Sky Survey (2MASS) Source Catalog (Skrutskie et al. 2006). We computed the plate solution using the IRAF task ccmap and the root-mean-square (rms) residuals obtained from ccmap are typically less than few hundredths of an arcsecond in both R.A and Decl. Moreover, the total alignment error, computed by summing the X-ray and optical rms residuals in quadrature, are much smaller than the X-ray positional uncertainties. Thus, we continued the search for optical counterparts to the transient sources using the positional uncertainties quoted in the Table 2

\section{ANALYSIS AND RESULTS}

\subsection{Transient Source Selection}

Transient X-ray sources were identified by visual inspection as well as comparing the final source lists from the 2001 and 2010 XMM-Newton observations. We selected the sources which 'disappeared' or 'appeared' in the long, $127 \mathrm{ks}$ XMM-Newton observation and classified them as transient candidate (TC) or possible transient candidate (PTC). These sources were detected in at least one observation with an unabsorbed $0.3-8 \mathrm{keV}$ luminosity of $>1 \times 10^{36} \mathrm{erg} \mathrm{s}^{-1}$ at a $>4 \sigma$ confidence level and not detected in another observation. In addition, we calculated the luminosity ratios between the "on-state" (the peak X-ray luminosities) and "off-state" (the nondetection upper limits) for these sources to confirm their transient nature. We classified the sources with the ratios $>5$ as TCs and those with the ratios between 1 and 5 as PTCs. The flux ratio used here is slightly different from the value used (high/low ratios $\sim 8$ ) in the Chandra studies of transient sources in M33 (Williams et al. 2008). The classification leads to the detection of 15 TCs and PTCs in NGC 55, listed in Table 2.

SRW06 identified $42 \mathrm{X}$-ray sources in the $\mathrm{D}_{25}$ region of NGC 55 using the XMM1 and XMM2 observations. We identified nine more sources in the XMM3 observation. Out of nine sources, two (XMMU J001548.1-391612 and XMMU J001604.6-391538) of them were not cataloged in SRW06, but detected in the XMM1 and XMM2 observations. Thus these two sources do not satisfy our criteria and are not included in the analysis. The remaining seven sources in XMM3 were again verified by performing the source detection procedure in the $0.3-1 \mathrm{keV}, 1-2 \mathrm{keV}$, and $2-6 \mathrm{keV}$ images. These sources were detected with a $4 \sigma$ threshold $(\mathrm{mlmin}=10)$ in at least one of the three energy bands. Moreover, they were cataloged in the third generation XMM-Newton Serendipitous Source Catalog (3XMM-DR4) 1 and flagged as the sources detected with high quality (summary flag $\leq 1$, where a low summary flag value indicates a high quality for a detection[2]). In addition, there are eight sources identified in SRW06 but not detected in the XMM3 observation (See Table 21). In total, our classification identified six TCs and nine PTCs in NGC 55. Among them, T1, whose properties have been reported in JW15 in detail, is likely a transient

1 http://xmmssc-www.star.le.ac.uk/Catalogue/3XMM-DR4/

2 http://xmmssc-www.star.le.ac.uk/Catalogue/2XMM/UserGuide_x black hole XRB in the star-forming region of NGC 55 . For completeness, the source is also listed in this paper.

\subsection{Hardness Ratio and Variability}

Since transient sources have few net counts, hardness ratios (HRs) can be considered as a primary tool to investigate their spectral properties. Although we cannot conclusively classify an individual source based on its X-ray colors alone, however it is possible to identify the trend in the source population. Therefore, the HRs of the transients were calculated from the count rates, which are defined as $\mathrm{HR} 1=(\mathrm{M}-\mathrm{S}) /(\mathrm{M}+\mathrm{S})$ and $\mathrm{HR} 2=(\mathrm{H}-\mathrm{M}) /(\mathrm{H}+\mathrm{M})$, where $\mathrm{S}, \mathrm{M}$ and $\mathrm{H}$ are the count rates in soft $(0.3-1 \mathrm{keV})$, medium $(1-2 \mathrm{keV})$, and hard $(2-6 \mathrm{keV})$ bands respectively. For the sources detected in EPIC-PN and MOS camera, we quote the weighted mean of hardness ratios (weights were calculated using the count rates) and they are listed in Table 3 . The X-ray color classification scheme of Jenkins et al. (2005) developed for $X M M-N e w t o n$ was used to classify the transient sources. The scheme divides the X-ray sources into four broad categories: absorbed sources (ABSs; HR1 > 0.57), XRBs $(-0.24<\mathrm{HR} 1<0.57,-0.8<\mathrm{HR} 2<0.8)$, SNRs (HR1 $<-0.24$, HR2 $<-0.10)$ and background sources $($ HR $1<-0.24$, HR2 $>-0.10$; See Table 3 of Jenkins et al. (2005) for more details). If we combine the 'absorbed' and 'XRB' categories into a single 'XRB' category, as in SRW06, nine out of 15 sources fall in the XRB category. The remaining six sources are soft, and one of them, T10, falls under the SNR category.

Five transient sources were detected in the 2001 Chandra observation and we classified them using the color classification scheme of Kilgard et al. (2005), tuned for Chandra data (See Table 2 of Kilgard et al. (2005) for more details). The obtained HRs for these sources are consistent with those from the $X M M-N e w t o n$ observations.

The HR-luminosity diagram (hardness-intensity proxy) of the TCs/PTCs detected in NGC 55 is given in Figure 1 From the figure it is clear that the majority of the TCs/PTCs have the color consistent with those of the persistent XRBs in the $\mathrm{D}_{25}$ region of the galaxy. For six source, their HR values are softer than the persistent XRB population and X-ray luminosities are lower compared to the other transient candidates, which suggest that they are the soft source population in this galaxy.

For the transient sources T1 and T2, we extracted the background subtracted light curve based on the combined EPIC-PN and MOS camera over $0.3-8 \mathrm{keV}$ energy range. We investigated the short-term X-ray variability by performing the Kolmogorov-Smirnov (K-S) test on the light curves binned with 800-s, 300-s and 100-s. From the K-S test, we found that these sources showed a strong short-term X-ray variability at confidence level of $>99.99 \%$.

\subsection{Spectral Analysis}

X-ray spectra of the transient sources within the $\mathrm{D}_{25}$ region of NGC 55 were extracted in the $0.3-8 \mathrm{keV}$ band, using a circular extraction aperture with radius ranges from $12-20$ arcsec. The different extraction radii were ased. etbmlvoid the contamination from the nearby sources 
TABLE 2

Properties of the transient CANDidAte sourCes Detected in NGC 55

\begin{tabular}{|c|c|c|c|c|c|c|c|c|c|}
\hline $\begin{array}{l}\text { Src } \\
\text { No. }\end{array}$ & $\begin{array}{c}\text { Catalog } \\
\text { No. }\end{array}$ & ObsID & $\begin{array}{c}\text { R.A. } \\
\text { (h:m:s) }\end{array}$ & $\begin{array}{c}\text { Dec. } \\
\left({ }^{\circ}:^{\prime}:^{\prime \prime}\right)\end{array}$ & $\begin{array}{c}r_{1 \sigma} \\
(\operatorname{arcsec})\end{array}$ & $\begin{array}{c}\text { Extr. Rad } \\
\text { (arcsec) }\end{array}$ & $\begin{array}{c}\text { Net } \\
\text { Counts }\end{array}$ & Ratio & TC/PTC \\
\hline$\overline{\mathrm{T} 1}$ & T1(JW15) & XMM3 & $00: 14: 46.81$ & $-39: 11: 23.48$ & 0.54 & 14 & 2134 & 10.7 & $\overline{\mathrm{TC}}$ \\
\hline $\mathrm{T} 2$ & 75(SRW06) & XMM1 & $00: 15: 34.27$ & $-39: 14: 24.80$ & 0.52 & 15 & 3009 & 95.5 & $\mathrm{TC}$ \\
\hline T3 & 27 (SRW06) & $\mathrm{XMM} 2$ & $00: 14: 35.45$ & $-39: 11: 32.20$ & 1.50 & 13 & 66 & 1.3 & PTC \\
\hline $\mathrm{T} 4$ & 30(SRW06) & $\mathrm{XMM} 2$ & $00: 14: 37.18$ & $-39: 11: 22.00$ & 2.26 & 14 & 69 & 1.1 & PTC \\
\hline $\mathrm{T} 5$ & 37 (SRW06) & $\mathrm{XMM} 2$ & $00: 14: 43.74$ & $-39: 12: 41.50$ & 1.49 & 12 & 73 & 1.3 & PTC \\
\hline T6 & 44(SRW06) & XMM1 & $00: 14: 52.68$ & $-39: 12: 23.90$ & 1.26 & 20 & 103 & 4.1 & PTC \\
\hline $\mathrm{T} 7$ & - & XMM3 & $00: 14: 54.86$ & $-39: 14: 16.97$ & 1.79 & 20 & 87 & 1.0 & PTC \\
\hline $\mathrm{T} 8$ & 51(SRW06) & XMM1 & $00: 15: 00.62$ & $-39: 12: 16.40$ & 1.16 & 14 & 95 & 1.8 & PTC \\
\hline T9 & 53(SRW06) & XMM1 & $00: 15: 03.79$ & $-39: 14: 59.80$ & 1.52 & 15 & 45 & 6.5 & TC \\
\hline $\mathrm{T} 10$ & - & XMM3 & $00: 15: 13.74$ & $-39: 13: 27.12$ & 1.65 & 15 & 154 & 1.6 & PTC \\
\hline T11 & - & XMM3 & $00: 15: 21.64$ & $-39: 16: 12.46$ & 2.16 & 13 & 105 & 1.7 & PTC \\
\hline $\mathrm{T} 12$ & - & XMM3 & $00: 15: 43.89$ & $-39: 17: 55.22$ & 0.96 & 15 & 136 & 5.9 & $\mathrm{TC}$ \\
\hline $\mathrm{T} 13$ & - & XMM3 & $00: 15: 52.85$ & $-39: 16: 34.39$ & 1.81 & 20 & 94 & 5.0 & $\mathrm{TC}$ \\
\hline T14 & - & XMM3 & $00: 16: 11.32$ & $-39: 16: 37.05$ & 1.20 & 15 & 188 & 5.8 & TC \\
\hline $\mathrm{T} 15$ & 122(SRW06) & XMM1 & $00: 16: 19.34$ & $-39: 16: 45.50$ & 2.61 & 15 & 57 & 3.9 & PTC \\
\hline
\end{tabular}

Note. - (1) Source number used in this paper; (2) Source identification number in JW15 and SRW06; (3) Observation ID in which the source was detected; (4)-(5) Right Ascension (R.A.) and Declination (Dec.) of each source (J2000.0); (6) 1 $\sigma$ positional uncertainty of the source; (7) Extraction radius; (8) Net counts from the EPIC-PN and MOS in 0.3-8 keV; (9) Luminosity Ratio; (10) Source Classification as TC or PTC (see text for details).

TABLE 3

HARDNESS RATIOS AND LUMINOSITIES OF TRANSIENT CANDIDATES

\begin{tabular}{cccccc}
\hline $\begin{array}{c}\text { Src } \\
\text { No. }\end{array}$ & ObsID & HR1 & HR2 & Class & $\mathrm{L}_{\mathrm{X}}$ \\
\hline T1 & XMM3 & $0.55 \pm 0.03$ & $-0.37 \pm 0.02$ & XRB & $37.76_{-0.03}^{+0.03}$ \\
T2 & XMM1 & $0.31 \pm 0.01$ & $-0.09 \pm 0.01$ & XRB & $38.22_{-0.02}^{+0.02}$ \\
& C-2255 & $0.54 \pm 0.01$ & $-0.06 \pm 0.01$ & XRB & $38.23_{-0.03}^{+0.03}$ \\
T3 & XMM2 & $-0.78 \pm 0.30$ & $0.27 \pm 0.93$ & SOFT & $36.42_{-0.30}^{+0.40}$ \\
T4 & XMM2 & $-0.70 \pm 0.26$ & $-0.17 \pm 0.29$ & SOFT & $36.16_{-0.35}^{+0.20}$ \\
T5 & XMM2 & $0.66 \pm 0.35$ & $-0.31 \pm 0.09$ & ABS & $36.52_{-0.22}^{+0.21}$ \\
& C-2255 & $0.55 \pm 0.11$ & $-0.31 \pm 0.07$ & XRB & $36.55_{-0.18}^{+0.18}$ \\
T6 & XMM1 & $0.76 \pm 0.50$ & $-0.16 \pm 0.05$ & ABS & $37.15_{-0.19}^{+0.34}$ \\
& C-2255 & $0.91 \pm 0.14$ & $-0.11 \pm 0.02$ & ABS & $36.62_{-0.14}^{+0.15}$ \\
T7 & XMM3 & $-0.68 \pm 1.70$ & $0.08 \pm 1.59$ & SOFT & $36.10_{-0.19}^{+0.14}$ \\
T8 & XMM1 & $0.18 \pm 0.07$ & $-0.13 \pm 0.17$ & XRB & $36.94_{-0.20}^{+0.23}$ \\
& C-2255 & $0.39 \pm 0.09$ & $0.30 \pm 0.05$ & XRB & $36.79_{-0.13}^{+0.13}$ \\
T9 & XMM1 & $-0.69 \pm 0.30$ & $-0.33 \pm 0.31$ & SOFT & $36.43_{-0.20}^{+0.20}$ \\
T10 & XMM3 & $-0.51 \pm 0.22$ & $-0.73 \pm 1.26$ & SNR & $36.12_{-0.34}^{+0.26}$ \\
T11 & XMM3 & $0.61 \pm 0.78$ & $0.29 \pm 0.12$ & ABS & $36.94_{-0.58}^{+2.71}$ \\
& C-2255 & $0.64 \pm 0.15$ & $0.33 \pm 0.07$ & ABS & $37.90_{-1.23}^{+1.52}$ \\
T12 & XMM3 & $0.14 \pm 0.02$ & $-0.16 \pm 0.03$ & XRB & $36.74_{-0.17}^{+0.38}$ \\
T13 & XMM3 & $0.56 \pm 0.22$ & $0.06 \pm 0.01$ & ABS & $36.59_{-0.25}^{+0.91}$ \\
T14 & XMM3 & $-0.74 \pm 0.10$ & $-0.76 \pm 0.43$ & SOFT & $36.42_{-0.12}^{+0.12}$ \\
T15 & XMM1 & $0.39 \pm 0.25$ & $-0.30 \pm 0.15$ & XRB & $36.70_{-0.38}^{+2.10}$ \\
\hline
\end{tabular}

Note. - (1) Source number; (2) Observation ID, where C indicates the Chandra observation used in the analysis; (3)-(4) Hardness Ratios derived from the count rate (See §3.2); (5) Nature of the source according to the classification scheme of Jenkins et al. (2005) and Kilgard et al. (2005); (6) Unabsorbed 0.3-8 keV X-ray luminosity in units of $\mathrm{erg} \mathrm{s}^{-1}$ derived from best-fit single component model.

in the crowded region. Background spectra were extracted from nearby source-free regions, with the same aperture radius. We used the SAS task ESPECGET to obtain source and background spectra together with the ancillary response file (ARF) and redistribution matrix file $(\mathrm{RMF})$ required in the spectral analysis. We binned the data to a minimum of 20 counts per bin and in many cases $\chi^{2}$ minimization was used to fit the data. In other cases where there were no sufficient spectral

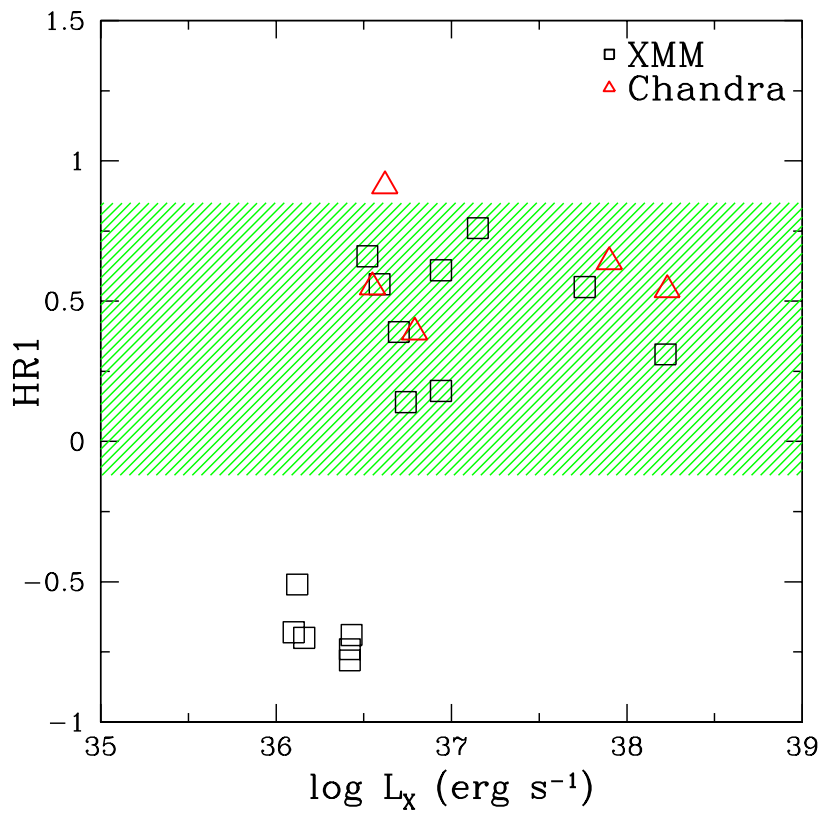

FIG. 1.- Hardness ratio versus X-ray luminosity for the transient candidates in NGC 55. The uncertainties are not plotted in order to have a clear view of the data points, which are given in Table 3 The shaded region indicates the range of persistent X-ray binary sources in the $\mathrm{D}_{25}$ region of the galaxy. The X-ray luminosities were estimated from best-fit single component models (see Section 3.3

counts, the Cash Statistic (Cash 1979) was used for spectral fitting. Because the Cash Statistic does not provide a goodness-of-fit measure like $\chi^{2}$, we additionally performed 5000 Monte Carlo simulations of each spectrum using the Goodness task (Arnaud 1996) to evaluate the quality of the fit for Cash Statistic. The fit is considered to be acceptable, if the "goodness" is $\leq 50 \%$. For $\chi^{2}$ statistic, we used the null hypothesis probability to check the quality. The spectra were fitted in the XSPEC version 12.8.1g (Arnaud 1996). The unabsorbed fluxes were derived using the convolution model CFLUX of XSPEC and the X-ray luminosities were estimated by assuming 
the distance of 1.78 Mpc (Karachentsev et al. 2003). All errors quoted were computed at a $90 \%$ confidence level.

We initially fitted both EPIC-PN and MOS spectra simultaneously using single-component models such as power law (PL) and multicolor disc blackbody (DISKBB). In all cases, the preferred model is the model with the lowest $\chi^{2} /$ d.o.f. However, favoring the spectral models for the low counting statistics sources is difficult, hence we report only the absorbed power law model parameters for these sources. An intervening absorption column (TBABS) was also applied to each model, which includes the Galactic column density towards NGC 55. If the best-fit value of $\mathrm{N}_{\mathrm{H}}$ is below the Galactic absorption, we froze $\mathrm{N}_{\mathrm{H}}$ to the Galactic value, $1.72 \times 10^{20} \mathrm{~cm}^{-2}$ (Dickey \& Lockman 1990). The spectral fitting results are summarized in Table 4. For the majority (13 out of 15 sources) of the sources a power law provides a marginally or significantly better fit than a disk blackbody model.

We estimated the upper limits on the count rates using EREGIONANALYSE task in SAS for the sources that were not detected in the XMM-Newton observations. After accounting for the background, the $90 \%$ confidence upper limits in $0.3-8 \mathrm{keV}$ energy band were derived. Assuming for each source the best-fit spectral model derived from the observations with detection, the flux upper limits were also obtained.

Source T2 has 3009 and 1781 net counts detected in the $X M M-N e w t o n$ and Chandra observations respectively. A single-component model, absorbed power law, provided an acceptable fit (See Figure 2) with $\Gamma=1.71 \pm 0.09$, $\chi^{2} /$ d.o.f $=183.5 / 150$ for the XMM-Newton and $\Gamma=$ $1.56 \pm 0.13, \chi^{2} /$ d.o.f $=77.1 / 70$ for the Chandra observations. The implied $0.3-8 \mathrm{keV}$ luminosity from our best-fit model is $1.70_{-0.11}^{+0.12} \times 10^{38} \mathrm{erg} \mathrm{s}^{-1}$. We also attempted a two-component model fitting, power law plus disc blackbody, for T2. The two-component model provided a marginal improvement to the spectral fit, with $\Delta \chi^{2} \sim 4$ for two extra degrees of freedom, over the single-component fit. The best fit yielded power-law index $\Gamma=1.79 \pm 0.15$ plus $0.14_{-0.03}^{+0.09} \mathrm{keV}$ disc blackbody, absorbed by $(0.36 \pm 0.16) \times 10^{22} \mathrm{~cm}^{-2}$, with $\chi^{2} /$ d.o.f $=179.8 / 148$ and the unabsorbed luminosity is $L_{X}=2.74_{-1.01}^{+3.15} \times 10^{38} \mathrm{erg} \mathrm{s}^{-1}$. The marginal improvement over the single component fit indicates that the presence of the second component is tentative, and we do not have a good constraint on the flux contribution from the disc. The estimated disc blackbody component contribution to the total $0.3-8 \mathrm{keV}$ source flux is $<35 \%$.

Barnard et al. (2014) successfully classified the X-ray transient sources in the galaxy M31 using the double thermal model (disc blackbody and blackbody), which was proposed by Lin et al. (2007) to examine the spectral evolution of transient LMXBs. Barnard et al. (2014) identified $36 \mathrm{BH}$ candidates that exhibit apparent hard state spectra with luminosities much higher than that for NS LMXBs. Using the double thermal model parameters, they found that none of the $\mathrm{BH}$ candidates occupied in the NS LMXB region. We also tested the double thermal model for T2 and the spectral fit is not significantly improved $\left(\chi^{2} /\right.$ d.o.f $=182.9 / 149, \Delta \chi^{2} \sim 1$ for one extra degree of freedom) over the absorbed power law model, but the spectral parameters, $k T_{\text {in }}=0.54 \pm 0.12$

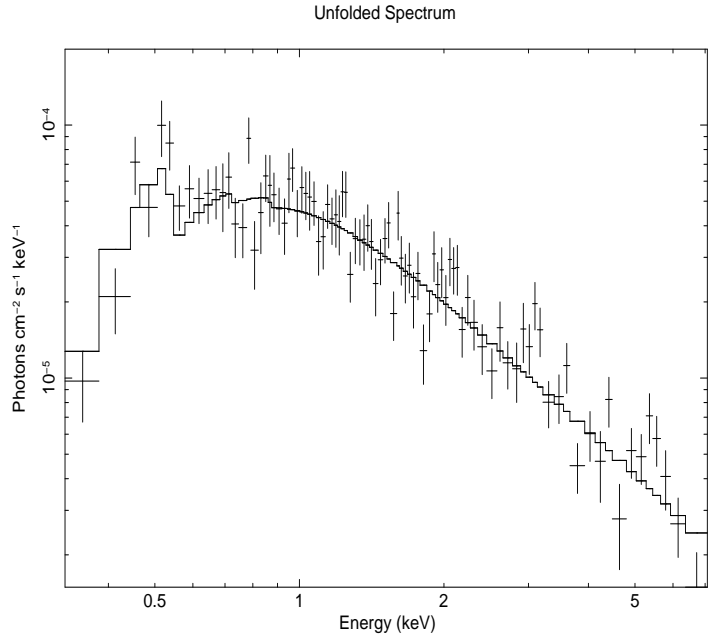

Fig. 2.- XMM-Newton EPIC-PN $0.3-8 \mathrm{keV}$ spectrum of T2, fitted with an absorbed power law model.

$\mathrm{keV}, k T_{B B}=1.30_{-0.15}^{+0.29} \mathrm{keV}$, and $L_{X}(2-10 \mathrm{keV})=$ $1.2_{-0.55}^{+0.66} \times 10^{37} \mathrm{erg} \mathrm{s}^{-1}$ are consistent with the case of $\mathrm{BH}$ candidates.

Seven sources (T5, T6, T8, T11, T12, T13, and T15) have spectra marginally or well fitted with the absorbed power law with $\Gamma \sim 1.6-2.4$ and the model luminosities are in the range of $\sim 3.3-14.1 \times 10^{36} \mathrm{erg} \mathrm{s}^{-1}$. For the disc blackbody model, the inner disc temperature obtained ranges from $\sim 1.2-2.9 \mathrm{keV}$ for the sources T11, T12 and T13. In most of the cases, the values of $\mathrm{N}_{\mathrm{H}}$ obtained higher than the Galactic foreground column density towards NGC 55. These spectral analysis suggests that these source are consistent with being NS and $\mathrm{BH}$ binaries in either a hard state or a thermal dominated state (Remillard \& McClintock 2006).

In the section 3.2 , we found that source T10 had HRs consistent with those of SNRs. We fitted its spectrum with a thermal plasma model, APEC in XSPEC. The spectrum is best-fitted with a thermal plasma temperature of $0.17_{-0.13}^{+0.24} \mathrm{keV}$, a solar metal abundance and an absorption of $0.77_{-0.51}^{+1.15} \times 10^{22} \mathrm{~cm}^{-2}\left(\chi^{2} /\right.$ d.o.f $\left.=18.5 / 20\right)$. The estimated unabsorbed $0.5-2 \mathrm{keV}$ X-ray luminosity is $\sim 3.3 \times 10^{37} \mathrm{erg} \mathrm{s}^{-1}$. We also tried the blackbody (BBODY) model for this source and the obtained parameters are given in Table 5.

The sources that are classified as soft sources by the HRs (T3, T4, T7, T9 and T14) have spectra favored by the power law as well as disc blackbody model. In all cases, the power law model fits yielded steep power law indices $(\Gamma \sim 3.4-5.6)$, reflecting the soft nature of the emission. Moreover, the inner disc temperature of these sources obtained from the disc blackbody model fits are much softer $\left(k T_{i n} \sim 0.1-0.12 \mathrm{keV}\right)$ than that of the typically observed Galactic XRBs $\left(k T_{i n} \sim 0.7-2 \mathrm{keV}\right)$. For these source, we tested the BBODY model and the spectral parameters are given in Table 5. The spectral fits obtained from the BBODY model are very similar to the power law or disc blackbody, except for T14. For the source T14, the BBODY fit is worse compared to the power law (See Figure 3). The temperature yielded from the BBODY model for these sources are in the range of $\sim 50-180 \mathrm{eV}$, consistent with that of SSSs, and the $\mathrm{X}$-ray luminosities are $\sim 10^{36} \mathrm{erg} \mathrm{s}^{-1}$. 
TABLE 4

BeST-Fit PARAMETERS OF THE SOURCE SPECTRA

\begin{tabular}{|c|c|c|c|c|c|c|c|c|c|c|c|c|}
\hline Src & & & & $\mathrm{PL}^{\mathrm{a}}$ & & & & DISKBB $^{a}$ & & & & \\
\hline No & $\operatorname{ObsID}^{b}$ & $\mathrm{~N}_{\mathrm{H}}^{c}$ & $\Gamma^{d}$ & $\log \mathrm{L}_{\mathrm{X}}{ }^{e}$ & $\chi^{2} /$ d.o.f ${ }^{f}$ & $\mathrm{G}^{\mathrm{g}}$ & $\mathrm{N}_{\mathrm{H}}^{c}$ & $\mathrm{kT}_{\mathrm{in}}{ }^{h}$ & $\log \mathrm{L}_{\mathrm{X}}{ }^{e}$ & $\chi^{2} /$ d.o.f ${ }^{f}$ & $\mathrm{G}^{\mathrm{g}}$ & $\log \mathrm{LXUP}^{i}$ \\
\hline$\overline{\mathrm{T} 1}$ & XMM3 & $0.68_{-0.06}^{+0.07}$ & $3.15_{-0.17}^{+0.18}$ & $38.38_{-0.10}^{+0.11}$ & $110.7 / 105$ & 0.33 & $0.31_{-0.04}^{+0.04}$ & $0.72_{-0.05}^{+0.05}$ & $37.76_{-0.03}^{+0.03}$ & $97.4 / 105$ & 0.69 & $\overline{<36.73}$ \\
\hline $\mathrm{T} 2$ & XMM1 & $0.19_{-0.03}^{+0.03}$ & $\begin{array}{l}1.71_{-0.09}^{+0.09} \\
\end{array}$ & $38.22_{-0.02}^{+0.02}$ & $183.5 / 150$ & 0.03 & $0.04_{-0.02}^{+0.02}$ & $1.58_{-0.11}^{+0.12}$ & $38.10_{-0.02}^{+0.02}$ & $210.2 / 150$ & 0.001 & $<36.24$ \\
\hline & C-2255 & $0.19_{-0.06}^{+0.06}$ & $1.56_{-0.13}^{+0.13}$ & $38.23_{-0.03}^{+0.03}$ & $71.4 / 70$ & 0.43 & $0.03_{-0.03}^{+0.04}$ & $1.79_{-0.19}^{+0.23}$ & $38.12_{-0.03}^{+0.02}$ & $77.1 / 70$ & 0.26 & \\
\hline T3 & XMM2 & $0.0172(f)$ & $4.29_{-1.49}^{+2.68}$ & $36.42_{-0.30}^{+0.40}$ & $6.4 / 6(\mathrm{C})$ & $27 \%$ & & & & & & $<36.32$ \\
\hline $\mathrm{T} 4$ & XMM2 & $0.0172(f)$ & $3.40_{-0.98}^{+1.68}$ & $36.16_{-0.35}^{+0.20}$ & $9.3 / 7(\mathrm{C})$ & $44 \%$ & & & & & & $<36.12$ \\
\hline T5 & XMM2 & $0.0172(f)$ & $1.69_{-0.55}^{+0.60}$ & $36.52_{-0.22}^{+0.21}$ & $7.3 / 6(\mathrm{C})$ & $37 \%$ & & & & & & $<36.41$ \\
\hline & $\mathrm{C}-2255$ & $0.0172(f)$ & $1.51_{-0.72}^{+0.85}$ & $36.55_{-0.18}^{+0.18}$ & $4.4 / 4(\mathrm{C})$ & $22 \%$ & & & & & & \\
\hline T6 & XMM1 & $0.24_{-0.24}^{+0.42}$ & $1.71_{-0.81}^{+1.23}$ & $37.15_{-0.19}^{+0.18}$ & $14.4 / 13(\mathrm{C})$ & $32 \%$ & & & & & & $<36.54$ \\
\hline & C-2255 & $0.0172(f)$ & $1.69_{-0.77}^{+0.96}$ & $36.62_{-0.14}^{+0.15}$ & $5.6 / 5(\mathrm{C})$ & $21 \%$ & & & & & & \\
\hline $\mathrm{T} 7$ & XMM3 & $0.0172(f)$ & $4.82_{-1.23}^{+1.76}$ & $36.17_{-0.19}^{+0.14}$ & $32.5 / 25$ & 0.15 & $0.0172(f)$ & $0.10_{-0.03}^{+0.05}$ & $36.10_{-0.19}^{+0.14}$ & $31.5 / 25$ & 0.17 & $<36.09$ \\
\hline T8 & XMM1 & $0.10_{-0.10}^{+0.31}$ & $1.61_{-0.59}^{+1.00}$ & $36.94_{-0.20}^{+0.23}$ & $10.7 / 11(\mathrm{C})$ & $16 \%$ & & & & & & $<36.69$ \\
\hline & C-2255 & $0.0172(f)$ & $0.90_{-0.46}^{+0.45}$ & $36.79_{-0.13}^{+0.13}$ & $4.3 / 8(\mathrm{C})$ & $6 \%$ & & & & & & \\
\hline Т9 & XMM1 & $0.0172(f)$ & $5.64_{-1.76}^{+3.70}$ & $36.43_{-0.20}^{+0.20}$ & $4.5 / 11(\mathrm{C})$ & $1 \%$ & & & & & & $<35.62$ \\
\hline $\mathrm{T} 10$ & XMM3 & $0.0172(f)$ & $2.08_{-0.75}^{+1.10}$ & $36.12_{-0.34}^{+0.20}$ & $17.0 / 21$ & 0.71 & $0.0172(f)$ & $0.45_{-0.23}^{+0.46}$ & $35.93_{-0.28}^{+0.20}$ & $17.1 / 21$ & 0.71 & $<35.92$ \\
\hline T11 & XMM3 & $2.50_{-1.94}^{+4.20}$ & $2.36_{-1.56}^{+3.41}$ & $36.94_{-0.58}^{+2.34}$ & $6.1 / 9$ & 0.73 & $1.62_{-1.28}^{+2.94}$ & $1.61_{-0.98}^{+0.00}$ & $36.54_{-0.30}^{+0.39}$ & $6.6 / 9$ & 0.68 & $<36.72$ \\
\hline & C-2255 & $3.82_{-2.23}^{+5.05}$ & $3.42_{-2.32}^{+1.30}$ & $37.90_{-1.23}^{+1.58}$ & $2.5 / 3(\mathrm{C})$ & $11 \%$ & & & & & & \\
\hline $\mathrm{T} 12$ & XMM3 & $0.32_{-0.21}^{+0.31}$ & $2.08_{-0.71}^{+1.13}$ & $36.74_{-0.17}^{+0.38}$ & $8.7 / 11$ & 0.65 & $0.12_{-0.12}^{+0.19}$ & $1.21_{-0.53}^{+0.98}$ & $36.54_{-0.16}^{+0.14}$ & $9.5 / 11$ & 0.58 & $<35.97$ \\
\hline $\mathrm{T} 13$ & XMM3 & $0.48_{-0.44}^{+0.99}$ & $1.51_{-1.17}^{+2.38}$ & $36.59_{-0.25}^{+0.17}$ & $26.9 / 14$ & 0.02 & $0.27_{-0.25}^{+0.67}$ & $2.86_{-2.30}^{+0.00}$ & $36.53_{-0.31}^{+0.16}$ & $27.6 / 14$ & 0.02 & $<35.89$ \\
\hline T14 & XMM3 & $0.0172(f)$ & $3.78_{-0.91}^{+1.17}$ & $36.42_{-0.12}^{+0.12}$ & $12.0 / 10$ & 0.28 & $0.0172(f)$ & $0.12_{-0.04}^{+0.09}$ & $36.31_{-0.16}^{+0.14}$ & $16.7 / 10$ & 0.08 & $<35.66$ \\
\hline $\mathrm{T} 15$ & XMM1 & $0.27_{-0.27}^{+1.35}$ & $2.08_{-1.21}^{+5.73}$ & $36.70_{-0.38}^{+2.10}$ & $0.6 / 3(\mathrm{C})$ & $1 \%$ & & & & & & $<36.11$ \\
\hline
\end{tabular}

Note. — ${ }^{a}$ Spectral models used for fitting: PL—power law continuum; DISKBB - multi-color disc blackbody. ${ }^{b}$ Observations used in each fit. ${ }^{c}$ Absorption column density, including Galactic absorption, in units of $10^{22} \mathrm{~cm}^{-2}$. ${ }^{d}$ Power law index. ${ }^{e}$ Logarithmic unabsorbed 0.3-8 keV X-ray luminosity in units of $\mathrm{erg} \mathrm{s}^{-1}$, calculated by assuming the distance of $1.78 \mathrm{Mpc}$ (Karachentsev et al. 2003). ${ }^{f}$ The $\chi^{2} /$ d.o.f value for the model, If $\mathrm{C}$-statistics is adopted, it is indicated with a C. ${ }^{g}$ Goodness of fit (Goodness when using Cash-statistics and null hypothesis probability for $\chi^{2}$ statistics). ${ }^{h}$ Inner disc temperature in units of keV. ${ }^{i}$ Upper limit on the X-ray luminosity for non-detection.

TABLE 5

SPECTRAL PARAMETERS FOR SUPER SOFT SOURCES USING BBODY MODEL

\begin{tabular}{lccccr}
\hline Src No. & $\mathrm{N}_{\mathrm{H}}$ & $\mathrm{kT}$ & $\log \mathrm{L}_{\mathrm{X}}$ & $\chi^{2} /$ d.o.f & \multicolumn{1}{c}{$\mathrm{G}$} \\
\hline T3 & $1.72(f)$ & $120_{-38}^{+51}$ & $36.17_{-0.26}^{+0.22}$ & $4.9 / 6$ & $13 \%$ \\
$\mathrm{~T} 4$ & $1.72(f)$ & $176_{-45}^{+52}$ & $36.08_{-0.25}^{+0.17}$ & $4.4 / 7$ & $6 \%$ \\
$\mathrm{~T} 7$ & $1.72(f)$ & $78_{-23}^{+30}$ & $36.08_{-0.19}^{+0.15}$ & $31.4 / 25$ & 0.18 \\
T9 & $1.72(f)$ & $50_{-20}^{+44}$ & $36.41_{-0.26}^{+0.24}$ & $6.2 / 11$ & $2 \%$ \\
T14 & $1.72(f)$ & $90_{-22}^{+37}$ & $36.31_{-0.16}^{+0.14}$ & $17.5 / 10$ & 0.06 \\
T10 & $1.72(f)$ & $229_{-85}^{+190}$ & $35.83_{-0.27}^{+0.19}$ & $18.0 / 21$ & 0.65 \\
\hline
\end{tabular}

Note. - (1) Source number; (2) Absorption column density in $10^{20} \mathrm{~cm}^{-2}$; (3) Black body temperature in $\mathrm{eV}$; (4) Logarithmic unabsorbed $0.3-8 \mathrm{keV}$ X-ray luminosity in $\mathrm{erg} \mathrm{s}^{-1}$; (5) The $\chi^{2} /$ d.o.f values for the model; (6) Goodness of fit.

We note that among the $154 \mathrm{X}$-ray sources in NGC 55 identified by BWF15, five sources (T2, T5, T6, T8 and T11) of ours were also listed. The rest of the transient sources were not included in BWF15 and half of them were detected in the 2010 XMM-Newton observation. We compared the results of these five sources with BWF15 and their hardness ratio classification are consistent with our results. They have studied the spectral properties of X-ray sources with $>50$ net counts from Chandra data and only one source (T2) is listed in our transient catalog. Their best-fit model for this source is an absorbed power law and the best-fit parameters are $N_{H}=0.15 \pm 0.06 \times$ $10^{22} \mathrm{~cm}^{-2}$ and $\Gamma=1.5 \pm 0.1$ (the unabsorbed $\mathrm{L}_{\mathrm{X}}$ in 0.3 $8 \mathrm{keV}$ band is $\left.\sim 2 \times 10^{38} \mathrm{erg} \mathrm{s}^{-1}\right)$, consistent with that of ours. They have identified long-term variations for four sources listed in this work (T2, T5, T6 and T11) from Chandra and XMM-Newton observations, which are confirmed by our analysis presented here.
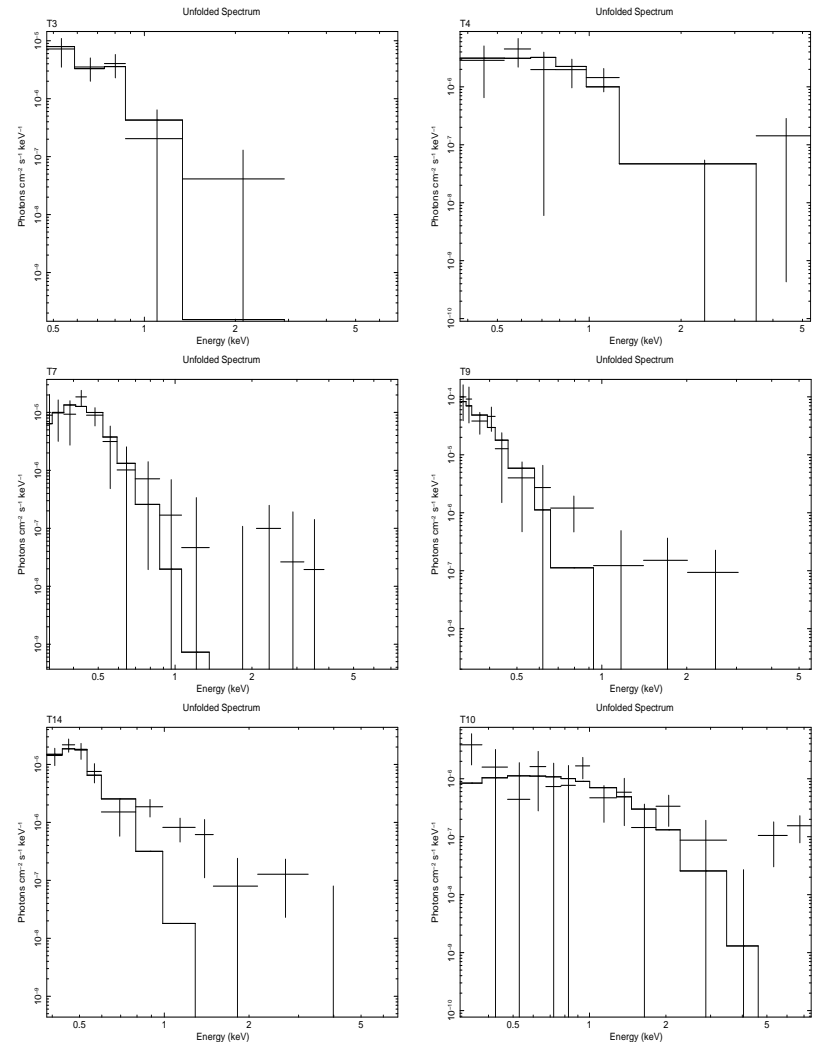

Fig. 3.- XMM-Newton EPIC-PN $0.3-8 \mathrm{keV}$ spectra of candidate soft sources (T3, T4, T7, T9, T14 and T10), fitted with an absorbed blackbody model. The blackbody fit becomes worse compared to the power law for T14. Most of them have only a little or no emission above $2 \mathrm{keV}$. 


\subsection{Optical Analysis and Comparison with Multi-wavelength Catalogs}

The transient sources were cross-correlated with the multi-wavelength catalogs available in the NED3, VizieR 4 , and SIMBAD 5 data bases. We searched these catalogs for matches within the $3 \sigma$ error radius of each $X M M-N e w t o n$ position. Of the 15 transient sources, source T15 positionally coincides with a galaxy, LCRS B001349.4-393325 (Shectman et al. 1996) and is likely a background AGN (SRW06). BWF15 reported the counterpart for T2 and T11 from GALEX GR6 data release. However, these $G A L E X$ sources are not in the $3 \sigma$ error circles of the XMM-Newton positions we have derived and hence we do not consider them associated to any of the X-ray sources.

We searched possible counterparts for the transient sources in the HST observations. Out of the 15 TCs/PTCs sources, seven are covered in the fields of view of $H S T$. For all these sources, the source region is crowded and the XMM-Newton error circle itself contains multiple optical sources. The HST fields are shown in Figure 4. We computed the magnitudes of sufficiently bright sources in each error circle using DAOPHOT (Stetson 1987) package in IRAF in F814W $(I)$ and $\mathrm{F} 606 \mathrm{~W}(V)$ bands. The magnitudes and colors, not corrected for reddening, of the brightest objects are given in Table 6 . The absolute $V$ magnitudes and $V-I$ colors of the optical sources have the values of $\sim[-0.2,-5.1]$ and $\sim[-0.8,1.5]$ respectively, which are broadly consistent with blue/red bright giants or supergiants, or with background AGNs. We also estimated the logarithmic X-rayto-optical flux ratio $\log \left(f_{x} / f_{o}\right)$, where $f_{x}$ is the $0.3-8 \mathrm{keV}$ flux and $f_{o}$ is the F606W flux.

In addition to the HRs and X-ray spectral fitting, we added X-ray-to-optical flux ratios and multi-wavelength information to characterize the possible nature of the transient sources. Table 6] summarizes the X-ray, optical and multi-wavelength observations available for each of the transient sources. Stars exhibit a wide range of flux ratio values, but typically $\log \left(f_{x} / f_{o}\right)<0$. For AGNs and BL Lac objects, their flux ratios are $>1$ (Maccacaro et al. 1982; Stocke et al. 1991). Moreover, the known accreting X-ray pulsars and HMXBs in the Small Magellanic Cloud (SMC) have a flux ratio $\lesssim 1$ with $B-V \lesssim 0$ (McGowan et al. 2008). If one of the optical sources in an errorcircle is the counterpart, the flux ratios would suggest that $\mathrm{T} 1$ and $\mathrm{T} 2$ are an $\mathrm{AGN}$, and rest of them are possible HMXBs. However, T1 exhibited outburst activity where accretion might occur only during certain parts of an orbit, which could fit in with a high mass donor star. Moreover, the colors of the optical sources in its error circle are not very blue in nature. If one of them is the counterpart of $\mathrm{T} 1$, it is more consistent with those of types A or F bright giant stars. Source T2 also has possible blue optical counterpart. BWF15 classified this source as an LMXB from their analysis using color-magnitude diagrams, X-ray color-magnitude diagrams, and the X-ray-to-optical flux ratio. Therefore the optical data suggest most of them are HMXBs, and

\footnotetext{
3 http://ned.ipac.caltech.edu/

4 http://vizier.u-strasbg.fr/viz-bin/VizieR/

5 http://simbad.u-strasbg.fr/simbad/
}

for $\mathrm{T} 1$ and $\mathrm{T} 2$, their overal properties favor XRB nature.

\section{DISCUSSION}

\subsection{BH and NS Candidates}

In addition to $\mathrm{T} 1$, which has been identified as a candidate BH XRB (JW15), T2 is another bright source and was detected in the XMM1, XMM2 and 2001 Chandra observations. However it completely disappeared in the long $127 \mathrm{ks} X M M$-Newton observation. The analysis showed that T2 changed its luminosity by at least two orders of magnitude from $\sim 10^{38}$ to $<10^{36} \mathrm{erg} \mathrm{s}^{-1}$. The HRs derived from the XMM-Newton and Chandra observations classified this source as an XRB system and it exhibited strong short-term variability in these observations. The spectrum of T2 is better described by an absorbed power law with $\Gamma \sim 1.7$, indicating that the source was likely in the hard state (Remillard \& McClintock 2006). The hard state is observed in BH and NS LMXBs (van der Klis 1994) only at luminosities $\leq 10 \%$ Eddington luminosity $L_{E d d}$ (Gladstone et al. 2007). Given its X-ray luminosity of $\sim 1.7 \times 10^{38} \mathrm{erg} \mathrm{s}^{-1}$ in $0.3-8 \mathrm{keV}$ band, which is $90 \% \mathrm{~L}_{\mathrm{Edd}}$ for a $1.4 \mathrm{M}_{\odot} \mathrm{NS}$, we suggest that T2 is likely a BH XRB exhibiting a hard state behavior during an outburst in 2001.

The canonical model for BH XRB sources, namely a combination of power law and disc blackbody components, marginally improved the spectral fit for T2. The resulting inner disc temperature is unusually low $(\sim 0.14$ $\mathrm{keV}$ ) indicating the presence of a cooler disc, which accounts for $<35 \%$ of the total observed X-ray flux. However, the $\Delta \chi^{2} \sim 4$ improvement for T2, for two extra degrees of freedom was not significant. Hence the presence of the soft component is tentative. The spectral modeling with double thermal components also marginally improves the fit compared to the single component model. For such a case, the analysis still suggests a BH system, since the disc blackbody temperature and $2-10 \mathrm{keV}$ disc blackbody luminosity of T2 do not fall in the NS LMXB parameter space.

Six sources (T5, T6, T8, T11, T12 and T13) are likely to be in either a hard state or a thermally dominated state. The X-ray luminosities of all these sources are $\leq 10 \%$ Eddington luminosity for either NS or BH primaries. This low accretion is observed in NS and $\mathrm{BH}$ $\mathrm{XRBs}$ in the hard state and suggests that the accretors of these binaries may be NS or BHs. However, there is no significant difference in the spectral fits and hence we cannot rule out a thermally dominated state from these low quality data. These sources could be NS or $\mathrm{BH} \mathrm{XRBs}$, and deeper observations are required for further classification.

\subsection{Super Soft Sources}

SSSs are classically defined as X-ray sources with temperature $\sim 100 \mathrm{eV}$ (Di Stefano \& Kong 2003) and typical $\mathrm{X}$-ray luminosities between $\sim 10^{36}$ and $10^{38} \mathrm{erg} \mathrm{s}^{-1}$. The steady nuclear burning of matter accreted onto the surface of a white dwarf (van den Heuvel et al. 1992) seems to be the most promising explanation for majority of these sources. SRW06 identified seven SSSs, which is one-sixth of total X-ray population in NGC 55. We identified five transient sources, which are soft sources on the basis of their HRs. Their soft emission is mod- 
TABLE 6

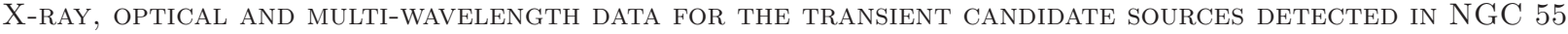

\begin{tabular}{|c|c|c|c|c|c|c|c|c|}
\hline $\begin{array}{l}\text { Src } \\
\text { No. }\end{array}$ & $\begin{array}{c}\text { HR } \\
\text { Class }\end{array}$ & Variability & $\begin{array}{c}\text { X-ray } \\
\text { Spectrum }\end{array}$ & $\mathrm{M}_{\mathrm{V}}$ & $V-I$ & $\begin{array}{c}\text { Range of } \\
V-I\end{array}$ & $\log \left(f_{x} / f_{o}\right)$ & $\begin{array}{l}\text { Multi- } \\
\text { wavelength }\end{array}$ \\
\hline$\overline{\mathrm{T} 1}$ & $\mathrm{XRB}$ & $\mathrm{LONG}$ & DISKBB & $\begin{array}{l}-2.64 \\
-2.33 \\
-1.93\end{array}$ & $\begin{array}{l}0.72(\mathrm{a}) \\
0.18(\mathrm{~b}) \\
0.62(\mathrm{c})\end{array}$ & {$[0.18,0.72]$} & {$[1.77,2.06](\mathrm{AGN})$} & - \\
\hline $\mathrm{T} 2$ & $\mathrm{XRB}$ & LONG & PL & $\begin{array}{l}-1.22 \\
-1.01 \\
-0.92\end{array}$ & $\begin{array}{c}0.55(\mathrm{a}) \\
-0.45(\mathrm{~b}) \\
0.46(\mathrm{c})\end{array}$ & {$[-0.82,1.05]$} & {$[2.79,3.20](\mathrm{AGN})$} & FUV? (BWF15) \\
\hline T3 & SOFT & - & - & $\begin{array}{l}-3.22 \\
-2.35 \\
-2.12\end{array}$ & $\begin{array}{c}-0.23(\mathrm{a}) \\
1.08(\mathrm{~b}) \\
0.55(\mathrm{c})\end{array}$ & {$[-0.23,1.08]$} & {$[0.18,0.71](\mathrm{HMXB})$} & - \\
\hline $\mathrm{T} 4$ & SOFT & - & - & $\begin{array}{l}-5.10 \\
-3.86 \\
-3.68\end{array}$ & $\begin{array}{c}0.99(\mathrm{a}) \\
0.48(\mathrm{~b}) \\
-0.38(\mathrm{c})\end{array}$ & {$[-0.40,1.46]$} & {$[-0.83,0.55](\mathrm{HMXB})$} & - \\
\hline T5 & ABS & LONG & - & - & - & - & - & - \\
\hline T6 & ABS & LONG & - & $\begin{array}{l}-3.45 \\
-2.93 \\
-2.25\end{array}$ & $\begin{array}{c}-0.15(\mathrm{a}) \\
0.79(\mathrm{~b}) \\
0.27(\mathrm{c})\end{array}$ & {$[-0.15,1.04]$} & {$[0.83,1.49]$ (HMXB?) } & - \\
\hline $\mathrm{T} 7$ & SOFT & - & BBODY & - & - & - & - & - \\
\hline $\mathrm{T} 8$ & XRB & - & - & $\begin{array}{l}-2.84 \\
-2.40 \\
-2.37\end{array}$ & $\begin{array}{c}0.79(\mathrm{a}) \\
0.09(\mathrm{~b}) \\
-0.10(\mathrm{c})\end{array}$ & {$[-0.10,0.79]$} & {$[0.86,1.36]$ (HMXB?) } & - \\
\hline T9 & SOFT & - & - & - & - & - & - & - \\
\hline $\mathrm{T} 10$ & SNR & - & PL & $\begin{array}{l}-3.61 \\
-3.36 \\
-3.30\end{array}$ & $\begin{array}{l}0.77(\mathrm{a}) \\
0.79(\mathrm{~b}) \\
1.16(\mathrm{c})\end{array}$ & {$[-0.19,1.41]$} & {$[-0.27,0.58](\mathrm{HMXB})$} & - \\
\hline T11 & ABS & LONG & PL & - & - & - & - & FUV? (BWF15) \\
\hline T12 & $\mathrm{XRB}$ & - & PL & - & - & - & - & - \\
\hline T13 & ABS & - & PL & - & - & - & - & - \\
\hline $\begin{array}{l}114 \\
\mathrm{~T} 15 \\
\end{array}$ & XRB & - & $\begin{array}{c}\text { PL } \\
- \\
\end{array}$ & - & - & - & - & Optical (SRW06) \\
\hline
\end{tabular}

Note. - (1) Source number; (2) Source classification based on the HRs; (3) Observed X-ray variability; (4) Best-fit X-ray spectral model when $\chi^{2}$ statistic is used; (5)-(6) The absolute $V$ magnitudes and $V-I$ colors of the brightest objects inside the $X M M$-Newton error circle and their labels are given in bracket; (7) Range of $V-I$ color of the optical sources in the error circle; (8) Range of logarithmic X-ray-to-optical flux ratio of the optical sources and the likely class given in bracket; (9) Multi-wavelength information available from the literature (See the text for more details).
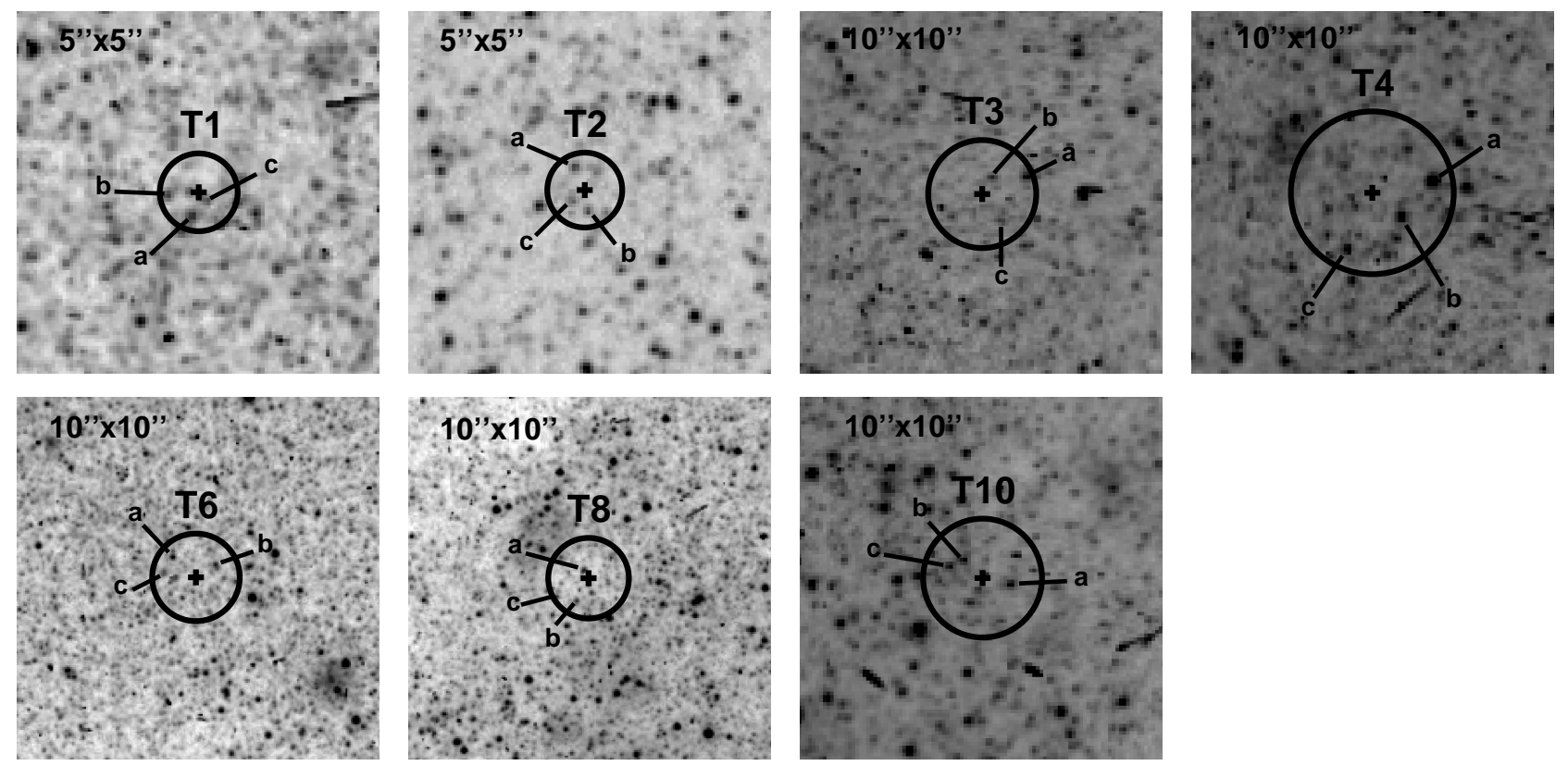

FIG. 4.- HST V-band images for the transient X-ray sources in NGC 55. The box size $\left(5^{\prime \prime} \times 5^{\prime \prime}\right.$ or $\left.10^{\prime \prime} \times 10^{\prime \prime}\right)$ is given in the left-top corner of each image. The black circle shows the $1 \sigma$ positional error circle centered on the source position, indicated by the 'plus' sign. The three brightest objects inside the error circle were labeled as "a", "b", "c" and their absolute $V$ magnitude and color are given in Table 6 North is up, and east is to the left. 
eled by a simple absorbed blackbody with temperatures of $\sim 50-180 \mathrm{eV}$. The cool emission indicates that these sources are most likely WD systems. The HRs of three sources are consistent with those of "typical" SSSs. Due to the given transient nature of these sources, they are likely to be classical novae which are identified as the major class of SSSs in the galaxies M31 and M33 (Pietsch et al. 2005). The X-ray emission from these systems arises due to the episodic thermonuclear burning of matter on the surface of the WDs, which leads to the transient nature (Pietsch et al. 2007).

We found that only one source (T10) had HRs consistent with those of SNRs, although the uncertainties on the HRs, particularly on HR2, are quite large. If we consider the thermal plasma model as a "standard SNR" model, the best fit plasma temperature of $\sim 0.2 \mathrm{keV}$ is lower than that of the SNR candidates in M33 and the unabsorbed $0.5-2 \mathrm{keV}$ luminosity is higher than the luminosities observed for the SNRs in M33 (Long et al.|2010). The blackbody fit rather provided a meaningful result, with temperature $(k T \sim 230 \mathrm{eV})$ consistent with QSSs. The emission from QSSs is too hot to be from WDs and the alternative physical systems include NS or BH binaries (Di Stefano et al. 2010) and SNRs (Orio 2006).

\subsection{Possible Optical Counterparts}

We searched the optical fields of 7 transient sources that are available from $H S T$ observations, which provide constraints on the source types for them. Although each $X M M-N e w t o n$ source position contains multiple optical sources, if one of the sources is the counterpart, the Xray-to-optical flux ratios suggest most of the transients as HMXBs, which is supported by the classification based on all available data. Further $H S T$ observations of the fields can identify the true counterparts by detecting variables among the optical sources.

We also cross-correlated the sources with publicly available multi-wavelength catalogs. Three of them, T2, T11 and T15, has a counterpart identified in the multiwavelength data, but the positions for the first two are not consistent with that from our XMM-Newton analysis. T15 could be either a NS or a BH XRB based on the spectral properties, but it is optically identified as a background AGN from multi-wavelength catalogs. SRW06 estimated $10-15$ background objects in the $\mathrm{D}_{25}$ region of the galaxy. We note that some of the new sources identified in the long $127 \mathrm{ks}$ observation have a color consistent with the absorbed category and they may be background objects. We estimated the number of background AGNs in the $\mathrm{D}_{25}$ region of NGC 55 using the $\log N-\log S$ relation reported in Campana et al. (2001), and the chance probability for detecting a background AGN inside the $\mathrm{D}_{25}$ region is $\sim 26 \%$.

\subsection{Comparison to Other Galaxies}

From X-ray perspective, NGC 55 is a typical Magellanic-type dwarf galaxy, which has a similar structure to that of LMC (de Vaucouleurs 1961). The X-ray properties of NGC 55 (SRW06), such as the total mass, neutral hydrogen mass, star formation rate (SFR), diffuse emission component, and discrete point source luminosities, illustrate the striking similarity with other nearby Magellanic systems, NGC 4449
TABLE 7

COMPARISON OF TRANSIENT PROPERTIES OF NGC 55 WITH $\mathrm{MCs}$

\begin{tabular}{lcc}
\hline \multicolumn{1}{c}{ Property } & NGC 55 & MCs $^{\mathrm{a}}$ \\
\hline Number of Transients & 15 & 64 \\
Fraction of HMXBs & $27 \%$ & $58 \%$ \\
Fraction of SSSs & $33 \%$ & $25 \%$ \\
$\mathrm{~L}_{\mathrm{X}}\left(\mathrm{erg} \mathrm{s}^{-1}\right)$ & $0.15-16.6 \times 10^{37}$ & $5 \times 10^{34}-3 \times 10^{39}$ \\
$\mathrm{~L}_{\mathrm{X}}$ of SSSs $\left(\mathrm{erg} \mathrm{s}^{-1}\right)$ & $1.5-2.7 \times 10^{36}$ & $6.7 \times 10^{34}-3 \times 10^{39}$ \\
\hline
\end{tabular}

Note. _ ${ }^{a}$ Kahabka \& Pietsch (1996); Haberl \& Pietsch (1999a. b); Kahabka et al. (1999, 2008); Coe et al. (2012); Li et al. (2012) and references there in.

and LMC (Wang et al. 1991; Vogler \& Pietsch 1997; Summers et al. 2003). The bright X-ray sources in these galaxies are spatially associated with star forming regions and broadly follow the correlation between the $\mathrm{X}$ ray luminosity and SFR established for active systems (Ranalli et al. 2003).

The transient source population has been extensively studied in our Milky Way (Jain et al. 2001; Tomsick et al. 2005; McClintock \& Remillard 2006, and many others), but relatively little work has been conducted on the extragalactic siblings. Due to the proximity, most extragalactic studies of transient sources concentrated on the Magellanic Clouds (MCs; Kahabka \& Pietsch 1996; Coe et al. 2001) and M31 (Williams et al. 2006, and references therein). A known population of transient sources in the MCs include XRBs and SSSs (Kahabka et al. 2008; Haberl et al. 2012; Sturm et al. 2013). The sources identified as LMXB transients are rare in Magellanic Clouds, but the Small Magellanic Cloud hosts a large number of high-mass X-ray binaries with a possible B/Be type companion (Kahabka \& Pietsch 1996; Haberl \& Pietsch 2004). Approximately 25-35 SSSs (including candidates) are observed in MCs, which are associated with magnetic cataclysmic variables, close binary SSSs (likely white dwarf and Be X-ray binaries), and classical post-nova (Kahabka \& Haberl 2006; Kahabka et al. 2006, 2008; Sturm et al. 2013), implying that the SSS population is an essential component in the Magellanic systems. Such effort has not been taken for NGC 4449. In Table 7. we summarize the comparison of transient properties of NGC 55 with MCs. Since MCs are frequent targets of many different X-ray observatories, a large number of transient sources were identified with luminosity ranges of $\sim 10^{34}-10^{39} \mathrm{erg} \mathrm{s}^{-1}$ compared to NGC 55 . However, the fractions of transient types in NGC 55 differ by no more than a factor of three with MCs. The marginal difference in the fractions of transient types may be due to observational effects such as effective extinction and the luminosity threshold of the available observations. This difference could be better investigated through continuous monitoring observations of NGC 55 as it is done for the MCs. The similarity in the transient and SSS populations, along with the X-ray properties of NGC 55 with other nearby Magellanic systems mentioned above, supports NGC 55 as a typical Magellanic system.

\section{SUMMARY}

We have presented a study of the transient X-ray source population in the Magellanic-type galaxy NGC 55. We analyzed the archival XMM-Newton and Chandra 
observations of NGC 55 and identified 15 candidate transient sources in the $\mathrm{D}_{25}$ region of the galaxy. Their X-ray luminosities are in the range of $10^{36}$ to $\sim 10^{38} \mathrm{erg} \mathrm{s}^{-1}$. The high sensitivities of the archival observations indicate a flux change of 1-2 orders of magnitude for some of these sources. The X-ray colors of these sources suggest that the transient population is dominated by XRBs. We performed detailed spectral and timing analysis of the data for two bright transient X-ray sources in the $\mathrm{D}_{25}$ region of the galaxy. The strong short-term variability seen in the sources, and the X-ray colors support the speculation of their high X-ray luminosity arises from an XRB system with a BH as the primary accretor. Six sources have spectral properties consistent with NS and $\mathrm{BH}$ XRBs accreting at both normal and high accretion rate. The transient population also seems to contain a sizable SSS component, perhaps one-third of the detected transient sources. The soft emission from them indicates that they are like white dwarf binaries. Optical analysis of archival HST data provides constraints on the likely nature for the sources and most of them are possible HMXBs. The cross-correlation with multi-wavelength catalogs identified one source as a possible background object, which is classified optically as a galaxy. From the spectral analysis a variety of properties have been determined, revealing the heterogeneous nature of transient sources in NGC 55. Our study suggests that the Magellanic-type galaxies could be a potential factory of transients, and comparative studies with other Magellanic-type galaxies and continuous monitoring at X-ray (multi) wavelengths will help us understand the physical nature of various types of high-energy objects that comprise the transient population.

\section{ACKNOWLEDGEMENTS}

We thank the anonymous referee for the helpful comments and recommendations that improved this manuscript. This work has made use of data obtained from the High Energy Astrophysics Science Archive Research Center (HEASARC), provided by NASA's Goddard Space Flight Center. This research was funded by Chinese Academy of Sciences Presidents International Fellowship Initiative (CAS PIFI, Grant No. 2015PM059), and was supported in part by the Strategic Priority Research Program "The Emergence of Cosmological Structures" of the Chinese Academy of Sciences (Grant No. XDB09000000).

\section{REFERENCES}

Arnaud, K. A. 1996, in Astronomical Society of the Pacific Conference Series, Vol. 101, Astronomical Data Analysis Software and Systems V, ed. G. H. Jacoby \& J. Barnes, 17

Barnard, R., Garcia, M., \& Murray, S. S. 2013, ApJ, 772, 126

Barnard, R., Garcia, M. R., Primini, F., \& Murray, S. S. 2014, ApJ, 791, 33

Binder, B., Williams, B. F., Eracleous, M., et al. 2015, AJ, 150, 94

Brassington, N. J., Fabbiano, G., Zezas, A., et al. 2012, ApJ, 755, 162

Burrows, D. N., Hill, J. E., Nousek, J. A., et al. 2005, Space Sci. Rev., 120, 165

Campana, S., Moretti, A., Lazzati, D., \& Tagliaferri, G. 2001, ApJ, 560, L19

Cash, W. 1979, ApJ, 228, 939

Coe, M. J., Negueruela, I., Buckley, D. A. H., Haigh, N. J., \& Laycock, S. G. T. 2001, MNRAS, 324, 623

Coe, M. J., Haberl, F., Sturm, R., et al. 2012, MNRAS, 424, 282

Dahlem, M., Weaver, K. A., \& Heckman, T. M. 1998, ApJS, 118, 401

de Vaucouleurs, G. 1961, ApJ, 133, 405

Di Stefano, R., Kong, A., \& Primini, F. A. 2010, New A Rev., 54, 72

Di Stefano, R., \& Kong, A. K. H. 2003, ApJ, 592, 884

-. 2004, ApJ, 609, 710

Di Stefano, R., Kong, A. K. H., Greiner, J., et al. 2004, ApJ, 610, 247

Dickey, J. M., \& Lockman, F. J. 1990, ARA\&A, 28, 215

Feng, H., \& Soria, R. 2011, New A Rev., 55, 166

Gladstone, J., Done, C., \& Gierliński, M. 2007, MNRAS, 378, 13

Gutiérrez, C. M. 2013, A\&A, 549, A81

Gutiérrez, C. M., \& López-Corredoira, M. 2007, A\&A, 472, 87

Haberl, F., \& Pietsch, W. 1999a, A\&AS, 139, 277

-. 1999b, A\&A, 344, 521

-. 2004, A\&A, 414, 667

Haberl, F., Sturm, R., Ballet, J., et al. 2012, A\&A, 545, A128

Jain, R. K., Bailyn, C. D., Orosz, J. A., McClintock, J. E., \& Remillard, R. A. 2001, ApJ, 554, L181

Jenkins, L. P., Roberts, T. P., Warwick, R. S., Kilgard, R. E., \& Ward, M. J. 2005, MNRAS, 357, 401

Jithesh, V., \& Wang, Z. 2015, MNRAS, 448, 1973

Kahabka, P., \& Haberl, F. 2006, A\&A, 452, 431

Kahabka, P., Haberl, F., Pakull, M., et al. 2008, A\&A, 482, 237

Kahabka, P., Haberl, F., Payne, J. L., \& Filipović, M. D. 2006, A\&A, 458, 285

Kahabka, P., \& Pietsch, W. 1996, A\&A, 312, 919
Kahabka, P., Pietsch, W., Filipović , M. D., \& Haberl, F. 1999, A\&AS, 136,81

Karachentsev, I. D., Grebel, E. K., Sharina, M. E., et al. 2003, A\&A, 404, 93

Kaur, A., Henze, M., Haberl, F., et al. 2012, A\&A, 538, A49

Kilgard, R. E., Cowan, J. J., Garcia, M. R., et al. 2005, ApJS, 159,214

Li, K. L., Kong, A. K. H., Charles, P. A., et al. 2012, ApJ, 761, 99

Lin, D., Remillard, R. A., \& Homan, J. 2007, ApJ, 667, 1073

Long, K. S., Helfand, D. J., \& Grabelsky, D. A. 1981, ApJ, 248, 925

Long, K. S., Blair, W. P., Winkler, P. F., et al. 2010, ApJS, 187, 495

Maccacaro, T., Gioia, I. M., Zamorani, G., et al. 1982, ApJ, 253, 504

McClintock, J. E., \& Remillard, R. A. 2006, Black hole binaries, ed. W. H. G. Lewin \& M. van der Klis, 157-213

McGowan, K. E., Coe, M. J., Schurch, M. P. E., et al. 2008, MNRAS, 383, 330

Monet, D. G. 1998, in Bulletin of the American Astronomical Society, Vol. 30, American Astronomical Society Meeting Abstracts, 120.03

Nooraee, N., Callanan, P. J., Barnard, R., et al. 2012, A\&A, 542, A120

Orio, M. 2006, ApJ, 643, 844

Pietsch, W., Fliri, J., Freyberg, M. J., et al. 2005, A\&A, 442, 879

Pietsch, W., Haberl, F., Sala, G., et al. 2007, A\&A, 465, 375

Pintore, F., Esposito, P., Zampieri, L., Motta, S., \& Wolter, A. 2015, MNRAS, 448, 1153

Ranalli, P., Comastri, A., \& Setti, G. 2003, A\&A, 399, 39

Read, A. M., Ponman, T. J., \& Strickland, D. K. 1997, MNRAS, 286,626

Remillard, R. A., \& McClintock, J. E. 2006, ARA\&A, 44, 49

Schlegel, E. M., Barrett, P., \& Singh, K. P. 1997, AJ, 113, 1296

Shectman, S. A., Landy, S. D., Oemler, A., et al. 1996, ApJ, 470, 172

Skrutskie, M. F., Cutri, R. M., Stiening, R., et al. 2006, AJ, 131, 1163

Stetson, P. B. 1987, PASP, 99, 191

Stiele, H., Pietsch, W., Haberl, F., et al. 2011, A\&A, 534, A55

Stobbart, A.-M., Roberts, T. P., \& Warwick, R. S. 2004, MNRAS, 351, 1063

-. 2006, MNRAS, 370, 25

Stocke, J. T., Morris, S. L., Gioia, I. M., et al. 1991, ApJS, 76, 813

Strüder, L., Briel, U., Dennerl, K., et al. 2001, A\&A, 365, L18 
Sturm, R., Haberl, F., Pietsch, W., et al. 2013, A\&A, 558, A3 Summers, L. K., Stevens, I. R., Strickland, D. K., \& Heckman, T. M. 2003, MNRAS, 342, 690

Tanaka, Y., \& Shibazaki, N. 1996, ARA\&A, 34, 607

Tomsick, J. A., Corbel, S., Goldwurm, A., \& Kaaret, P. 2005, ApJ, 630, 413

Turner, M. J. L., Abbey, A., Arnaud, M., et al. 2001, A\&A, 365, L27 van den Heuvel, E. P. J., Bhattacharya, D., Nomoto, K., \& Rappaport, S. A. 1992, A\&A, 262, 97

van der Klis, M. 1994, ApJS, 92, 511

Vogler, A., \& Pietsch, W. 1997, A\&A, 319, 459

Wang, Q., Hamilton, T., Helfand, D. J., \& Wu, X. 1991, ApJ, 374,475

Williams, B. F., Naik, S., Garcia, M. R., \& Callanan, P. J. 2006 ApJ, 643, 356

Williams, B. F., Gaetz, T. J., Haberl, F., et al. 2008, ApJ, 680, 1120 